\title{
Level of Readiness Index for Conceptual Design Refinement
}

\author{
Kenji Iino ${ }^{1, *}$, Masayuki Nakao ${ }^{2}$ \\ ${ }^{1}$ The University of Tokyo, Environmental Science Research Center, Tokyo 113-0033, Japan \\ ${ }^{2}$ The University of Tokyo, Graduate School of Engineering, Tokyo 113-8656, Japan
}

\begin{abstract}
The authors have been teaching conceptual design courses to graduate schools and adult groups. Despite the instructors' encouragement to refine a design to a level that clarifies the elemental functional requirement for each part element, the students often lump elemental functional requirements into higher level functions. The resulting Design Record Graph shows a functional requirement with multiple arcs extending to the corresponding nodes in the design parameter space. When such a design maps to a Design Matrix in Axiomatic Design, the matrix turns into a rectangular one with non-diagonal elements. Instead of just speaking to the students that they will face difficulty when it comes to producing prototypes, the authors developed a metric that quantifies the level of detail of a design so the students, often driven to gain higher numerical scores, will naturally spend efforts to refine their designs to levels that are ready for building prototypes. We call this metric Level of Readiness Index, i.e., LOR Index.
\end{abstract}

\section{Introduction}

The authors have been teaching creative design classes at several graduate schools in Japan; Sophia University (Sophia), Kyushu Institute of Technology (Kyutech), and The University of Tokyo (UTokyo). The one at Kyutech is a short 2-day course with a total of 9 hours. Both Sophia and UTokyo classes have 14 sessions each amounting to over 23 hours of class time. Classes at Sophia and Kyutech are offered in Japanese, whereas, the class at UTokyo is completely in English. A partial motivation for starting the English class at UTokyo was to enhance the students' communication skills in English for the coming years of globalization. The first year started as the instructors intended with a 50/50 split of Japanese and foreign students. As the years passed, however, despite the instructors' intention, the number of Japanese students kept dropping while that of foreign grew. Fig. 1 shows how the enrolment changed over the years. Whether the Japanese students shied away from the class because we conducted it in English or because of guiding the students to enhance their creativity is unknown. This is not a topic for this paper, however, it will be an interesting theme to look into in terms of motivating the students to study.

The students work in groups of 2 to 5 , whilst an ideal group number will be 4 , the students having to gather for homework and project completion sometimes force different size group formations. The short Kyutech class has no time for building prototypes, and the Sophia class, with the lack of proper workspace, also finishes with the groups presenting their design solutions using slides and turning in final reports. The UTokyo class also ends with final presentations, however, each group go through building a prototype, or as Roth [1] calls them "crap-up" as a simple and quick version of mock-up.

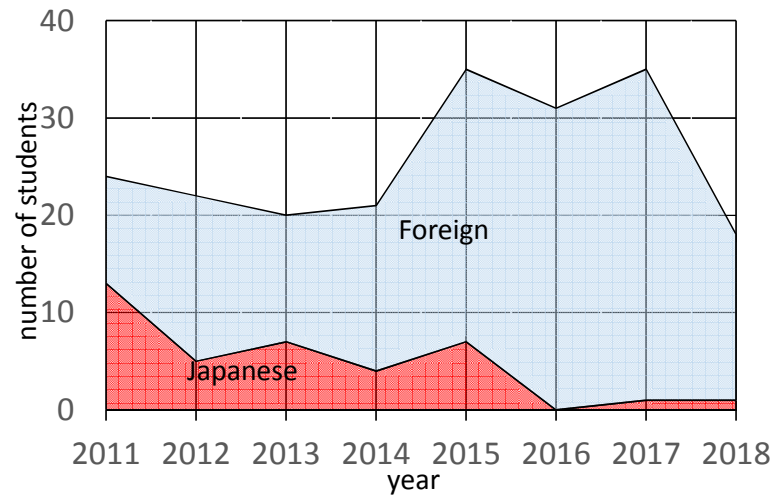

Fig. 1. Japanese and Foreign student enrolment over the years at UTokyo creative design class conducted in English

Although the class prototype building is simple, some of the student groups have to struggle in producing physical models that demonstrate their design concepts. Such difficulty is typical for a group that built a poor Design Record Graph (DRG) [2]. The authors, thus, concluded that a poor DRG is a sign of a conceptual design not being ready for proceeding to the next step. As the students polish up their DRGs, the instructors encourage them to produce better DRGs with further detail, however, as it is also a time for the students to start thinking about building their prototypes, their interest is directed more towards the fun part of hands-on model making.

Students, from their nature of looking for better scores and grades through their student lives, are naturally interested in quantitative evaluation than qualitative ones. We, thus, propose with this paper, a

\footnotetext{
Corresponding author: kiino@esc.u-tokyo.ac.jp
} 
quantitative index that evaluates how ready a conceptual design is by evaluating the DRG. We call this index the Level of Readiness (LOR) index. By directing the students' attention to this qualitative number, LOR index, they will work harder in the conceptual design stage. The efforts in terms of Axiomatic Design (AD) [3] is to bring the design closer to an uncoupled one in the conceptual stage.

\section{Design Record Graph and Axiomatic Design}

Our main design tool is the Design Record Graph (DRG) [2]. A DRG starts from the left with the Product Functional Requirement (PFR) which states the design goal of what function the design will offer to the customer. The DRG adopts the "divide and conquer" method by subdividing the PFR into smaller functional requirements (FRs) and repeating the subdivision until a set of functions unpractical for further subdivision is reached. We call these elemental FRs functional elements (FEs).

An FE in the functional space maps to physical elements (PEs) in the physical space. Fig. 2 shows a DRG with Liu's suggestion for the word "physical" instead of "structural" that we used in the 10th ICAD conference [2].

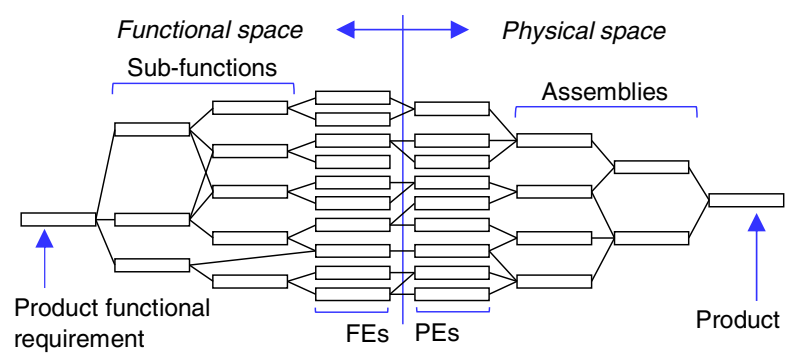

Fig. 2. Elements of a Design Record Graph (DRG)

When a designer constructs a DRG, however, the process will not strictly proceed from left to right. The designer's thinking process typically jumps from the functional space to the physical space at different levels. This manner of jumping is call zig-zagging [3].

The border between the functional and physical spaces of a DRG corresponds to the Design Matrix (DM) in $\mathrm{AD}$, i.e., the matrix $[\mathbf{A}]$ in eq. (1).

$$
\{\mathbf{F R}\}=[\mathbf{A}]\{\mathbf{D P}\}
$$

Liu and Lu showed design education using AD [4]. Although we do not teach AD in our classes, the efforts to reach a clear conceptual design that is ready for prototyping by targeting a one-to-one correspondence between the FEs and PEs is equivalent to aiming for an uncoupled design with $\mathrm{AD}$. If we reach an uncoupled design, the border at the functional and physical spaces will have the number of arcs, FEs and PEs all the same with horizontal arcs crossing the border each connecting an FE on the left to a PE on the right.

\section{Premature conceptual design}

The graduate students in the classes mostly have experience in mechanical design, however, rarely in creating a new design to meet the PFR that they defined by themselves. In this section, we will show a sample case which was a typical premature conceptual design proposed by a student group and relate the problems identified with this case to AD by showing what the DM would look like.

\subsection{Confusing parts and assemblies}

Fig. 3 shows a student sketch of a machine for remotely painting outside walls of high buildings and Fig. 4 is the DRG by the student group.

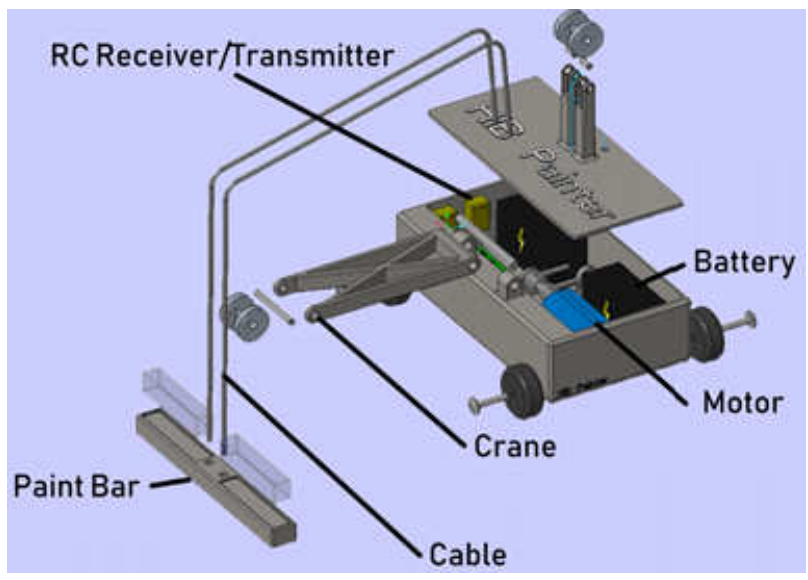

Fig. 3. Conceptual sketch of high building (bldg.) wall painter

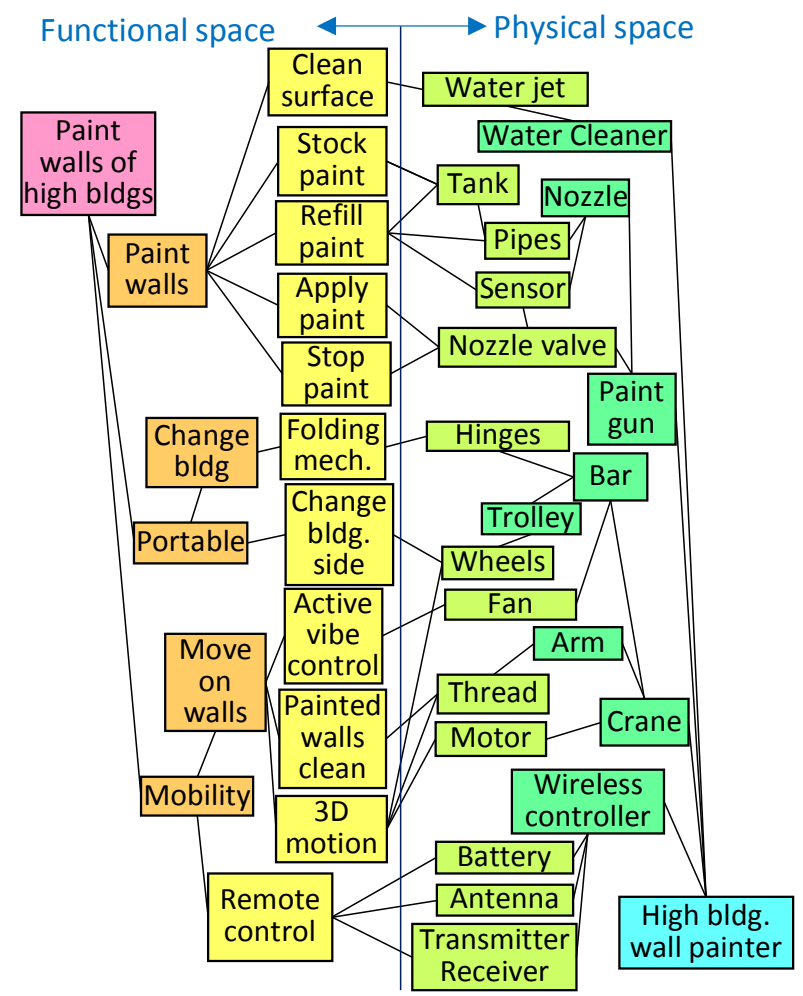

Fig. 4. DRG of high building wall painter 
The PFR is at the top left corner shown in pink and the corresponding product is at the bottom right corner in blue. FEs are shown in yellow and PEs in green.

A conceptual mistake common with students is confusing high level assemblies with parts in the physical space. FRs are at higher level in the functional space the more they are drawn to the left, whereas, in the physical space, higher level PEs are drawn to the right.

In Fig. 4 "Nozzle" is not a PE and it is drawn as an assembly of pipes and a sensor. We can guess the students designed the structure by first thinking "Nozzle to spray paint on the walls." Also, the students drew "Pipes" to transfer paint at a level higher than "Tank" to store the paint. As they came up with parts needed to realize the function of controlling the painting, they set them at levels lower than the original "Nozzle." We rearranged the partial DRG of the sub-function to "paint walls" as Fig. 5 shows. It has a nice ladder structure between the FEs and PEs meaning the design is uncoupled.

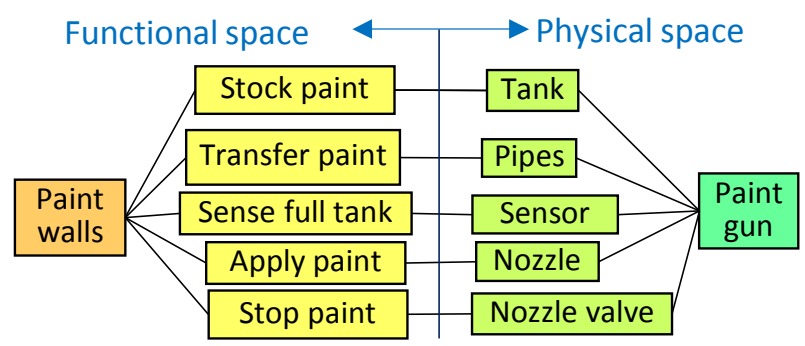

Fig. 5. Modified DRG of the sub-function "Paint walls"

\subsection{Realizing an FE with multiple PEs}

The second FE from the bottom in Fig. 4 is the FR to realize "3D motion." 3D motion in this case means to move the entire "High bldg. wall painter" along the edge of the roof, extend the "Crane arm" in and out using the "Thread" so the "Paint bar" with spray nozzles positions itself at the right distance from the wall, and the third movement, which actually the students left out from the DRG, is the vertical movement of the "Paint bar" by extending and retracting the "Cable."

The FE "3D motion," as Fig. 4 shows, corresponds to 3 PEs; "Wheels," "Thread," and "Motor." When we map this $1 \mathrm{FE}$ to 3 PEs correspondence to $\mathrm{AD}$, the $\mathrm{DM}$ will have a row with multiple Xs as Fig. 6 shows.

$$
\begin{aligned}
& \ldots \mathrm{X} \times \mathrm{X} \\
& \ldots
\end{aligned}
$$

Fig. 6. Row with multiple Xs in the DM (1FE to 3PE mapping)

The resulting design is a non-uncoupled (decoupled or coupled depending on other FE to PE mappings), however, the situation is not as bad as the multiple FEs to a single PE case this paper will discuss next.

This poorly configured design is a result of the designer not thinking about the real FE for each PE, in other words, the designer did not bother to look into the function of each physical part.

In their work, actually, the assignments proceeded in the sequence of first, developing the DRG, next, the sketch, and finally the prototype. It is likely that the students did not bother going back to the DRG to rework their findings into it because the "cable" shown in their sketch, Fig. 3, is missing from the DRG, Fig. 4.

Fig. 7 shows our rearrangement and addition of the DPs "Bar hanger" and "Cable" and their corresponding FRs missing from the student work. The rework goes to the physical space to add the obvious "Cable" and the motor to wind and release the cable, "Cable motor," phrasing their FRs, naming the two component assembly as "Bar hanger," and figuring out the phrase for the higher level FR "Move bar up/down" to lump the two PEs into one. The particular sequence for identifying the phrases for FRs and DPs may be different with the person reworking the detail, however, going back and forth between the functional and physical spaces at different levels shows how $\mathrm{AD}$ in the detail stage proceeds in a zig-zagging manner.

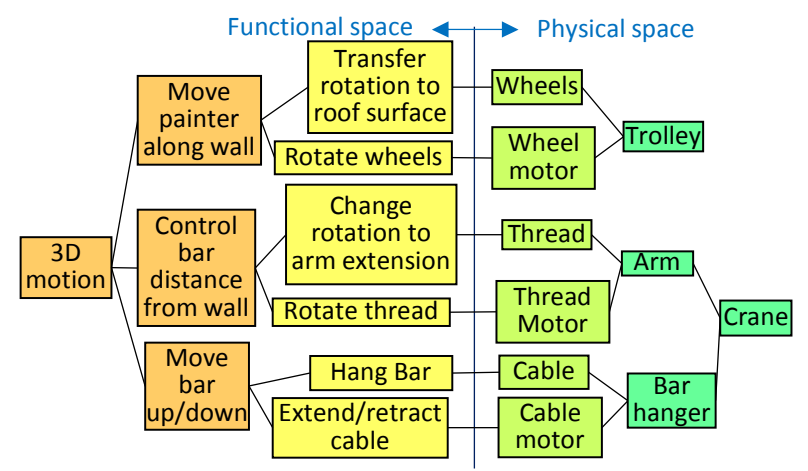

Fig. 7. Modified DRG of the sub-function " 3 D motion"

\subsection{Multiple FEs realized by a single PE}

Next, we turn our attention to the other case of a single PE realizing multiple FEs. In Fig. 4, the "Wheels" are used to satisfy 2 FEs, "Change bldg. side" and "3D motion." In Fig. 7 we modified the "3D motion" FE to a more refined one with the FE "Transfer rotation to roof surface" for the PE "wheels." The other FE for wheels in Fig. 4 is "Change bldg. side" meaning the entire painter will rotate about the vertical axis for 90 degrees, counterclockwise (CCW), (if the building is rectangular like in most cases) to paint a new wall.

This is a case that a design is really coupled. This interference will show in $\mathrm{AD}$ as a single column containing multiple Xs in the DM (Fig. 8).

$$
\begin{aligned}
& . \dot{X} . \\
& . X \\
& . .
\end{aligned}
$$

Fig. 8. Multiple Xs in 1 column in DM (2FE to 1PE mapping) 
The design is like turning a 4-wheel vehicle by 90 degrees and one can easily foresee that the action will result in a rather large radius in doing so and if the painter needs to paint the walls to the edges, it will probably have to move in the reverse direction to reach the end near the corner after making the turn.

Trying to fix this interference gives the designer a chance to think of a more creative solution. The question to ask is "How would I turn the painter without having to go around in a large radius?" If we search the net, we can see that spherical tires are under development $[5,6]$. For this case, however, the painter does not really need to be omnidirectional and if a large rotating column can push itself down to lift the entire painter unit, when it rotates and retracts, then the entire painter has completed a turn for an arbitrary angle on the spot (Fig. 9).

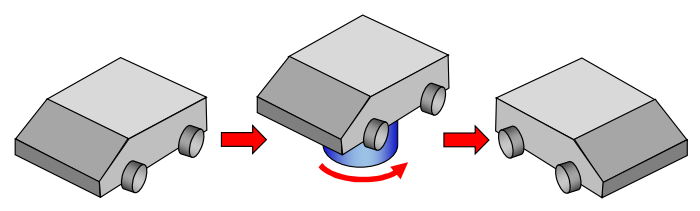

Fig. 9. Turning the painter by 90 degrees, $\mathrm{CCW}$, on the spot

The new design in Fig. 9 has a DRG shown in Fig. 10 with the new FE identified for the lifter/rotator.

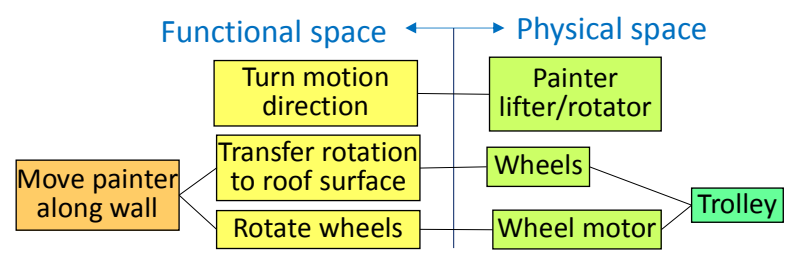

Fig. 10. Modified DRG of the mobility functions

\section{Level of readiness index}

Despite the authors' efforts and words to encourage the students to refine their DRGs, it is hard to see strong motivations in them to do so. It probably has to do with the qualitative nature of the task and difficulty in seeing the accomplishments quantitatively. To better motivate the students, the authors propose to define a quantitative evaluator of how fine a DRG is. We call it the Level Of Readiness index, i.e., LOR index.

When we have a DRG, we calculate the LOR index in the following manner.

(1) Evaluate all the PEs with the following criteria:

Assign 9 points to the PE when the designer can:

- Has or can easily produce a drawing of the part

- Knows a store or internet page for purchase

- Already has the part in hand

Assign 3 points to the $\mathrm{PE}$ when the designer can:

- Knows what the part looks like, however, still needs some design work (calculation, spatial planning, or testing) to decide the configuration

- Can draw a rough sketch

Assign 1 points to the PE when none of the above conditions are met.
The scores, 9, 3, and 1 are used for quality function deployment in determining specific part contribution to each customer value [7]. The logarithmic like scoring in 3 levels matches people's perception when they have to give scores to ambiguous concepts.

(2) Assign scores 9 to all the FEs

Unlike PEs that have shape, FEs describe functions to meet. Whether a stated function is concrete enough or is still too vague is difficult for people to assign scores. The penalty of not being well thought out is given in the PE evaluations.

(3) Divide the FE and PE scores by the number of arcs extending out to PEs for an FE and FEs for a PE.

The discussions in Section 3 showed why one to multiple correspondences are not welcome in conceptual design and how they map to interference in AD. Both FEs and PEs are penalized for having multiple arcs.

(4) For each arc that connects a FE and PE, calculate the average of the two element scores at its ends.

This step assigns a score to each FE-PE pair.

(5) For all the arcs that connect an FE to PE, sum the scores and divide by the number of such arcs. The result is the LOR index.

Depending on the design subject and the size of design, DRGs can have quite different sizes. The index shall not vary with the size of the DRG, i.e., the number of FE-PE pairs, thus the index simply calculates the average of them.

\subsection{LOR index for the DRG in Fig. 4}

This subsection shows the resulting LOR index from applying its calculation to the DRG in Fig. 4.

Fig. 11 is the calculation with only the FEs and PEs shown. On the left of FEs and right of PEs are the evaluator assigned scores. The PEs scored 3 because the design was not well detailed at this stage except 1 for "Fan" which was a mere idea. On the right of FEs and left of PEs are scores after the penalty of having multiple arcs. The number on an arc is the average of the two scores at its ends. The arc scores summed to 68 and the 17 arcs gave an LOR index of 4.0 for this design.

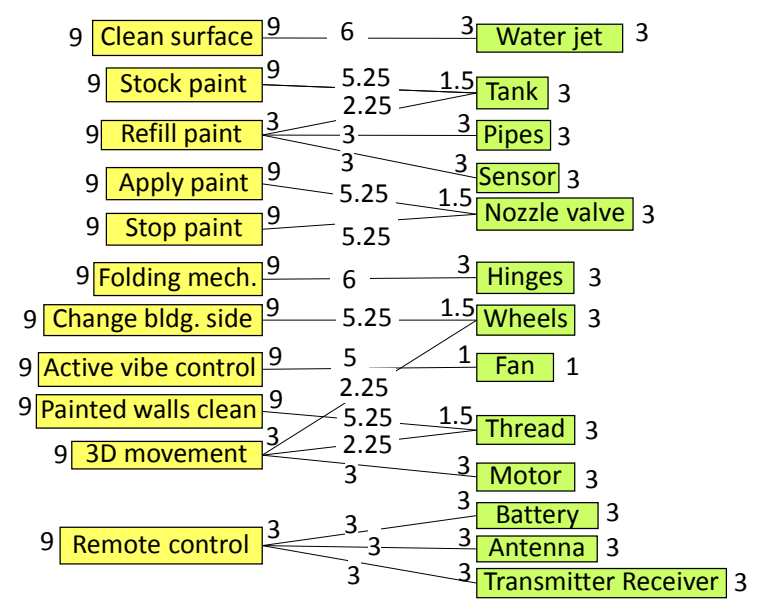

Fig. 11. LOR calculation for the DRG in Fig. 4 
Table 1. LOR calculation for the DRG in Fig. 4

\begin{tabular}{|c|c|c|c|c|c|c|}
\hline S(FE) & FE & S(Af) & $S(A)$ & $S(A p)$ & $\mathrm{PE}$ & $S(P E)$ \\
\hline 9 & Clean surface & 9 & 6 & 3 & Water jet & 3 \\
\hline 9 & Stock paint & 9 & 5.25 & 1.5 & \multirow{2}{*}{ Tank } & \multirow{2}{*}{3} \\
\hline \multirow{3}{*}{9} & \multirow{3}{*}{ Refill paint } & 3 & 2.25 & 1.5 & & \\
\hline & & 3 & 3 & 3 & Pipes & 3 \\
\hline & & 3 & 3 & 3 & Sensor & 3 \\
\hline 9 & Apply paint & 9 & 5.25 & 1.5 & \multirow{2}{*}{ Nozzle valve } & \multirow{2}{*}{3} \\
\hline 9 & Stop paint & 9 & 5.25 & 1.5 & & \\
\hline 9 & Folding mech. & 9 & 6 & 3 & Hinges & 3 \\
\hline 9 & Change bldg. side & 9 & 5.25 & 1.5 & \multirow{2}{*}{ Wheels } & \multirow{2}{*}{3} \\
\hline (9) & (3D movement) & 3 & 2.25 & 1.5 & & \\
\hline 9 & Active vibe control & 9 & 5 & 1 & Fan & 1 \\
\hline 9 & Painted walls clean & 9 & 5.25 & 1.5 & \multirow{2}{*}{ Thread } & \multirow{2}{*}{3} \\
\hline \multirow{2}{*}{9} & \multirow{2}{*}{ 3D movement } & 3 & 2.25 & 1.5 & & \\
\hline & & 3 & 3 & 3 & Motor & 3 \\
\hline \multirow{3}{*}{9} & \multirow{3}{*}{ Remote control } & 3 & 3 & 3 & Battery & 3 \\
\hline & & 3 & 3 & 3 & Antenna & 3 \\
\hline & & 3 & 3 & 3 & Transmitter/Reciever & 3 \\
\hline
\end{tabular}

Table 1 shows the tabulated form of the calculation. The evaluator assigns the score 9, 3, or 1 as $\mathrm{S}(\mathrm{FE})$ for each FE or S(PE) for each PE. Note that the FE "3D movement" occupies two places because one of its arc extended to the PE "Wheels" at a higher elevation in the DRG.

The remainder of the calculation is mechanical with each arc across the functional and physical spaces receiving $\mathrm{S}(\mathrm{Af})$, i.e., score of the left end of an arc;

$$
\mathrm{S}(\mathrm{Af}) \mathrm{i}=\frac{\mathrm{S}(\mathrm{FEi})}{\text { number of arcs from the i-th FE }}
$$

and $\mathrm{S}(\mathrm{Ap})$ for its right end;

$$
\mathrm{S}(\mathrm{Ap}) \mathrm{j}=\frac{\mathrm{S}(\mathrm{PEj})}{\text { number of arcs from the } \mathrm{j} \text {-th PE}}
$$

The score of an arc is the average of its scores at the two ends;

$$
S(A)=\frac{S(A f)+S(A p)}{2}
$$

and LOR is the average of the arc scores.

$$
\mathrm{LOR}=\frac{\Sigma \mathrm{S}(\mathrm{A})}{\text { number arcs across functional and physical spaces }}(4) \text {. }
$$

\subsection{LOR index for modified design in Section 3}

Now collect the modifications made in Section 3 and place them into the corresponding elements of the original design. The results are shown in Fig. 12 with the same convention for placing the scores. This time, the number of arcs is 18. The sum of the arc scores is 98 with an average of 5.4 .

Note that the calculation left the low score 1 for "Fan" not to give unreasonable advantage to the modified design. The new design also kept the poor configuration for the remote control that we did not show how to modify in Section 3. It is an easy task to identify the FEs for each of the three parts that make the "Remote control," however, we left it out.

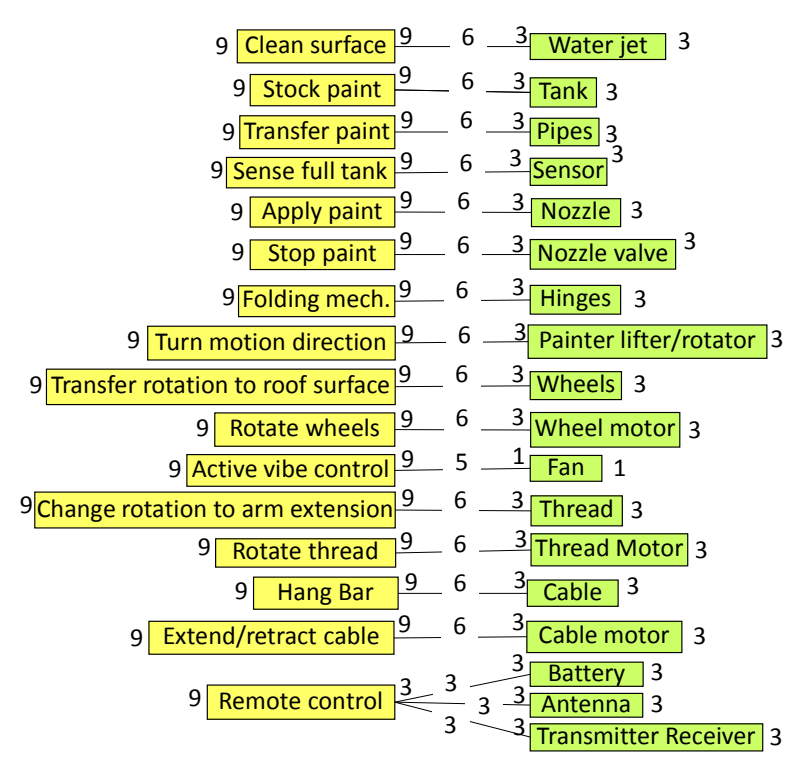

Fig. 12. LOR calculation for the modified design in Section 3

\section{Discussions}

Section 3 showed that as we work to identify an individual FE for each PE in the physical world, and devise a single PE for each FE without bundling multiple functions to burden one part, the design approaches an uncoupled one. This refinement process zig-zags across the functional and physical spaces because both FEs and PEs can be missing from a particular DRG, and whether the designer identifies a phrase for a FE or its corresponding PE depends on the designer.

Section 4 showed that the LOR index we propose is larger for a design that has gone through the refinement. The act of refining a design is equivalent to bringing it closer to an uncoupled design in AD.

The result in Section 4 had an increase in LOR from 4.0 to 5.4; an increase smaller than the authors expected before actually making the calculation. It is probably due to keeping all PEs at their stages of not being well designed. As the designer proceeds through further detail design, the LOR will increase.

The formulation in Section 4 leads to the best possible LOR index of 9 where each FE has its unique corresponding PE and all FEs receive 9 for being clear.

The worst possible LOR index is not 5, the average of 9 for the FE and 1 for the PE. As we saw in Table 1, multiple arcs extending out from FEs and PEs penalize the LOR. If we have all PEs reaching out to all FEs, the arcs receives scores on their left functional side divided by the number of FEs and their right physical side by the number of PEs. The LOR score approaches zero with more FEs and PEs laid out in such a manner.

It is rather easy for the students to see that multiple arcs radially extending out from a FE or entering into a single PE penalize the LOR. This motivates them to further split a FE with multiple arcs into a set of real elements that state the detail functions. In the latter case of multiple arcs entering a single PE they will try to split the PE into smaller parts, each corresponding to a single FE. The work is equivalent to modifying the design 
matrix so it approaches an uncoupled design. Reaching a further detailed design gives the designer a chance to enhance the entire design by identifying better ways, i.e., new PEs, to meet their real FEs.

Assigning points, 9, 3, or 1 to a FE is subjective and a more objective evaluation is possible by posing a set of questions for step (1) in Section 4. The following set of questions in a flow can give a fair evaluation:

$\begin{array}{cc}\text { Q1: Do you have the part? } & \text { Yes } \rightarrow \text { Assign } 9 \\ \text { No } & \\ \downarrow & \\ \text { Q2: Have a drawing of the part? } & \text { Yes } \rightarrow \text { Assign 9 } \\ \text { No } & \\ \downarrow & \\ \text { Q3: Know where to purchase the part? } & \text { Yes } \rightarrow \text { Assign 9 } \\ \text { No } & \\ \downarrow & \\ \text { Q4: Have a sketch of the part? } & \text { Yes } \rightarrow \text { Assign 3 } \\ \text { No } & \\ \downarrow & \\ \text { Assign 1 } & \end{array}$

\section{Conclusion}

This paper proposed Level Of Readiness (LOR) index to quantify how detail a design is. AD can interpret it to be a measure to tell the level of its approach to an uncoupled design. Having students calculate their LOR indexes at their design stages will encourage them to refine their DRGs before shifting all efforts into prototyping.

Future work with LOR will involve developing an automated application tool to calculate the LOR on the fly as the designer lays out the DRG. Thus, the tool better be able to lay out the DRG as the designer proceeds with conceptual design. It is not an easy task for the computer to keep track of hierarchies among nodes and at the same time remember and display the connections. Fig. 13 shows how a computer would draw the DRG in Fig. 2 with relatively small development efforts. By "relatively," the authors mean compared to a directed nodes and arcs graph diagram drawn freely on the $2 \mathrm{D}$ computer screen like in Fig. 2.

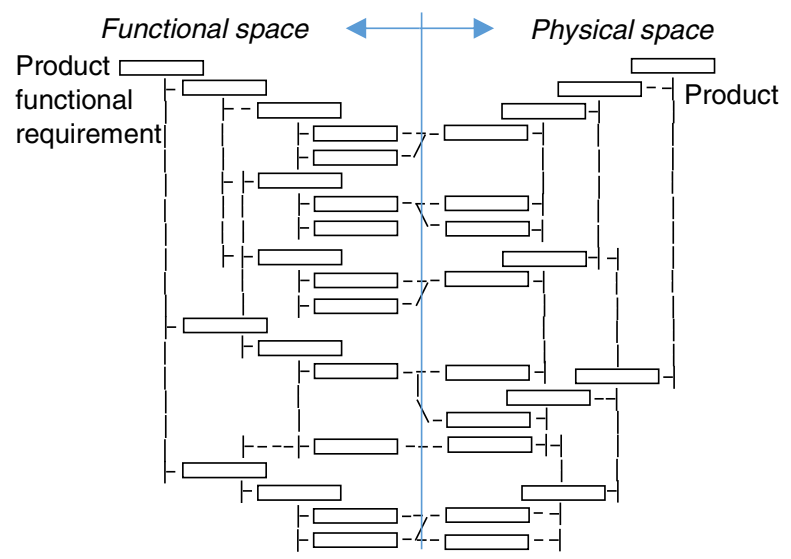

Fig. 13. How a computer would draw the DRG in Fig. 2
Comparing Fig. 13 and Fig. 2, one can see more structure-oriented depiction of the same information with Fig. 13. It will be easy to automatically calculate LOR from this structure, however, the strict hierarchical placing of functional and physical phrases interferes with the designer's creative mind. On the other hand, developing an application tool to handle nodes and arcs like in Fig. 2 is a large development. We may have to look into tools available for making such arc and node graph plots and carry out calculations of attributes given to such objects.

\section{References}

1. B. Roth, Design Process as an Educational Tool, Proceedings of the International Symposium of Mechanism and Machine Science, 2017

2. K. Iino, M. Nakao, Design Record Graph and Axiomatic Design for creative design education, PROCIR-D-16-00377, Proceedings of ICAD 2016

3. N. P. Suh, Axiomatic Design, Advances and Applications, Oxford University Press, 2001

4. A. Liu, S. Lu, Lessons learned from teaching axiomatic design in engineering design courses. Proceedings of ICAD 2013

5. F. Markus, Spherical tires : Might autonomous cards be borne on airless backetballs ?, May 9, 2017, Motortrend Homepage, viewed April 14, 2019, (https://www.motortrend.com/news/spherical-tiresautonomous-cars-borne-on-airless-basketballstechnologue/)

6. C. W. Wu, C. K. Hwang, A novel spherical wheel driven by Omni wheels, 2008 International Conference on Machine Learning and Cybernetics, IEEE

7. K. Ishii, K. Iino, Design for Manufacturing (in Japanese), Yokendo, Japan, 2008 